\title{
An improved BAC transgenic fluorescent reporter line for sensitive and specific identification of striatonigral medium spiny neurons
}

\author{
Kristen K. Ade ${ }^{1}$, Yehong Wan', Meng Chen ${ }^{1}$, Bernd Gloss ${ }^{2,3}$ and Nicole Calakos ${ }^{1,3 *}$ \\ Division of Neurology, Center for Translational Neuroscience, Duke University, Durham, NC, USA \\ 2 Neurotransgenic Core Facility, Duke University, Durham, NC, USA \\ ${ }^{3}$ Department of Neurobiology, Duke University, Durham, NC, USA
}

Edited by:

James M. Tepper, Rutgers, The State

University of New Jersey, USA

Reviewed by:

David M. Lovinger, National Institutes of Health, USA

Tibor Koos, Rutgers University, USA

${ }^{*}$ Correspondence:

Nicole Calakos, Center for Translational Neuroscience, Box 2900, Research Dr., Duke University Medical Center, Durham, NC 27710, USA.

e-mail:nicole.calakos@duke.edu
The development of BAC transgenic mice expressing promoter-specific fluorescent reporter proteins has been a great asset for neuroscience by enabling detection of neuronal subsets in live tissue. For the study of basal ganglia physiology, reporters driven by type 1 and 2 dopamine receptors have been particularly useful for distinguishing the two classes of striatal projection neurons - striatonigral and striatopallidal. However, emerging evidence suggests that some of the transgenic reporter lines may have suboptimal features. The ideal transgenic reporter line should (1) express a reporter with high sensitivity and specificity for detecting the cellular subset of interest and that does not otherwise alter the biology of the cells in which it is expressed, and (2) involve a genetic manipulation that does not cause any additional genetic effects other than expression of the reporter. Here we introduce a new BAC transgenic reporter line, Drd1atdTomato line 6, with features that approximate these ideals, offering substantial benefits over existing lines. In this study, we investigate the integrity of dopamine-sensitive behaviors and test the sensitivity and specificity of tdTomato fluorescence for identifying striatonigral projection neurons in mice. Behaviorally, hemizygous Drd1a-tdTomato line 6 mice are similar to littermate controls; while hemizygous Drd2-EGFP mice are not. In characterizing the sensitivity and specificity of line 6 mice, we find that both are high. The results of this characterization indicate that line 6 Drd1a-tdTomato ${ }^{+/}$mice offer a useful alternative approach to identify both striatonigral and striatopallidal neurons in a single transgenic line with a high degree of accuracy.

Keywords: medium spiny neuron, striatum, basal ganglia, direct pathway, striatonigral, BAC, transgenic, tdTomato

\section{INTRODUCTION}

The generation of transgenic lines with promoter-specific fluorescent reporter proteins has significantly advanced the field of neurobiological research. By allowing for cell subtype identification and morphological visualization in living tissue, BAC transgenics have greatly facilitated physiological studies of specific neuronal subsets. BAC transgenic mice generated by the Gene Expression Nervous System Atlas (GENSAT) project expressing EGFP fluorescent reporters under the control of the type 1 and 2 dopamine receptor regulatory sequences have been especially useful for discriminating between the two types of striatal projections neurons, striatonigral or direct pathway and striatopallidal or indirect pathway (Gong et al., 2003). These two types of medium spiny neurons (MSNs) have molecular, anatomical, and functional differences, but are not otherwise easily distinguished. Since the introduction of fluorescent reporter transgenic lines to distinguish these cell types in living tissue, they have been used widely to reveal phenotypic differences between these two classes of MSNs as well as evidence for subtype-selective impairment in disease states (Day et al., 2006, 2008; Kreitzer and Malenka, 2007; Shen et al., 2007, 2008; Ade et al., 2008; Cepeda et al., 2008; Gertler et al., 2008; Janssen et al., 2009; Yin et al., 2009; Doig et al., 2010; Flores-Barrera et al., 2010; Gittis et al., 2010; Planert et al., 2010; André et al., 2011).
While the GENSAT Drd1a-EGFP and Drd2-EGFP transgenic lines have been useful to study the physiology of striatopallidal and striatonigral MSNs, a recent study reports that that introduction of the BAC Drd2-EGFP transgene into the mouse genome may alter dopamine $\mathrm{D} 2$ receptor surface expression and cause aberrant locomotor behavior (Kramer et al., 2011). These findings raise the need for alternative methodologies to discriminate between these two populations of MSNs.

In a previous study, we evaluated the sensitivity and specificity of using a single transgenic fluorescent reporter to properly identify both populations of striatal projection neurons (Shuen et al., 2008). Fluorescence intensity of a transgenic reporter can vary across lines, limiting the utility of certain lines for identifying all cells expressing the fluorescent reporter, especially when using techniques with relatively low sensitivity such as epifluorescence microscopy commonly found on electrophysiological rigs. The development of the Drd1a-tdTomato line 5 (Shuen et al., 2008) was a significant advancement over the existing $D r d 1 a$-EGFP line as the td-Tomato red fluorescent protein has a higher relative brightness than EGFP (Deliolanis et al., 2008; Drobizhev et al., 2009) as well as a longer excitation wavelength which provides better tissue penetration. However, since its introduction, several characteristics that limit the robustness of the line 5 Drd1a-tdTomato transgenic 
line have been identified. Breeding experiments demonstrate an $\mathrm{X}$-linked inheritance pattern . In addition, ambiguous mammary glands are more commonly observed on male mice in this line. A putative X-linked transgene also adds another level of care that users of this line need to apply in experimental subject breedings in order to avoid inadvertently studying a biased population of only females in cases where the transgene donor is the father. Lastly, the fertility and litter-size of this transgenic line is severely reduced on a pure $\mathrm{C} 57 \mathrm{Bl} / 6$ strain background. Because $\mathrm{C} 57 \mathrm{Bl} / 6$ is a commonly used strain in neuroscience, this further limits the utility of this line.

In this study, we characterize a second Drdla-tdTomato BAC transgenic mouse line, line 6 . We find that this line has normal fertility in the $\mathrm{C} 57 \mathrm{Bl} / 6$ background, an autosomal-inheritance pattern, no obvious morphological or cellular abnormalities, normal behavior in dopamine-sensitive motor tests, and high sensitivity and specificity of the fluorescent reporter for identifying striatonigral MSNs. Altogether, these features overcome significant limitations of existing transgenic lines and provide a useful option for accurate discrimination of both striatonigral and striatopallidal neurons using a single transgenic line.

\section{MATERIALS AND METHODS MICE}

All animal procedures were done humanely to minimize distress and according to protocols approved by the Institutional Animal Care and Use Committee of Duke University. Mice used for behavioral experiments, Drd1a-tdTomato (line 6) and GENSAT Drd2-EGFP mice (courtesy of Drs. N. Heintz and X. W. Yang; provided to us from the Yang colony in 2006), were backcrossed onto a C57Bl/6 background for a minimum of six generations. In behavioral experiments, transgene-negative littermate controls $(-/-)$ were compared to mice that were hemizygous for the transgene $(+/-)$ of interest. Mice used for imaging experiments had hybrid strain backgrounds (sv129/FVB-1/C57Bl/6). ChAT-EGFP mice [B6.Cg-Tg(RP23268L19-EGFP)2Mik/J] were obtained from The Jackson Laboratory (Bar Harbor, ME, USA). Mice used for electrophysiology experiments were backcrossed onto a $\mathrm{C} 57 \mathrm{Bl} / 6$ background. All mice were offspring of breeding pairs in which transgene expression was limited to one parent ensuring that all experimental animals were hemizygous for transgenes of interest. Line 6 Drd1a-tdTomato mice are available to the academic research community through The Jackson Laboratory (JAX stock number 16204).

\section{GENOTYPING}

Genotyping of Drd1a-tdTomato was performed using the following primers: BGH-F (forward primer) 5-CTT CTG AGG CGG AAA GAA CC-3; dDR4 (reverse primer) 5-TTT CTG ATT GAG AGC ATT CG-3. The PCR protocol used is as follows: 1 cycle at $94^{\circ} \mathrm{C}$ for $2 \mathrm{~min}, 5$ cycles of $94^{\circ} \mathrm{C}$ for $30 \mathrm{~s}, 60-55^{\circ} \mathrm{C}$ touchdown ramp for $30 \mathrm{~s}$ and $72^{\circ} \mathrm{C}$ for $30 \mathrm{~s} ; 25$ cycles of $94^{\circ} \mathrm{C}$ for $30 \mathrm{~s}, 55^{\circ} \mathrm{C}$ for $30 \mathrm{~s}$ and $72^{\circ} \mathrm{C}$ for $30 \mathrm{~s}$ followed by $1 \mathrm{cycle}$ at $72^{\circ} \mathrm{C}$ for $5 \mathrm{~min}$. PCR product length is $846 \mathrm{bp}$. Genotyping of Drd2-EGFP was performed using the following primers: D2 (forward) CCC GAA GCT TCT CGA GGC GCG CCC TGT GCG TCA GCA TTT GGA GCA AC; GFPB2 (reverse) TCA GGG TCA GCT TGC CGT AGG5. The PCR protocol used is as follows: 1 cycle at $94^{\circ} \mathrm{C}$ for $2 \mathrm{~min}$,
5 cycles of $94^{\circ} \mathrm{C}$ for $30 \mathrm{~s}, 60-55^{\circ} \mathrm{C}$ touchdown ramp for $60 \mathrm{~s}$ and $72^{\circ} \mathrm{C}$ for $90 \mathrm{~s} ; 30 \mathrm{cycles}$ of $94^{\circ} \mathrm{C}$ for $30 \mathrm{~s}, 55^{\circ} \mathrm{C}$ for $60 \mathrm{~s}$ and $72^{\circ} \mathrm{C}$ for $90 \mathrm{~s}$ followed by 1 cycle at $72^{\circ} \mathrm{C}$ for $5 \mathrm{~min}$. PCR product length is approximately $800 \mathrm{bp}$. Genotyping of ChAT-EGFP was performed using the following primers: ChAT (forward) AGT AAG GCT ATG GGA TTC ATT C; ChAT (reverse) AGT TCA CCT TGA TGC CGT TC. The PCR protocol used is as follows: 1 cycle at $94^{\circ} \mathrm{C}$ for $3 \mathrm{~min}, 35$ cycles of $94^{\circ} \mathrm{C}$ for $30 \mathrm{~s}, 52^{\circ} \mathrm{C}$ for $60 \mathrm{~s}$ and $72^{\circ} \mathrm{C}$ for $60 \mathrm{~s}$ and $1 \mathrm{cycle}$ of $72^{\circ} \mathrm{C}$ for $2 \mathrm{~min}$. PCR product length is approximately $600 \mathrm{bp}$.

\section{BEHAVIOR}

Mice aged 8-11 weeks were tested in the open field (OF) behavioral assay. Animals in their home cages were moved to the room where OF testing was performed at least $12 \mathrm{~h}$ prior to the experiment. For testing, animals were placed in OF chambers $(21 \times 21 \times 30 \mathrm{~cm})$ with clear polycarbonate walls. VersaMax Animal Activity Monitor (AccuScan Instruments, Columbus, OH, USA) was used to record behavioral response parameters including horizontal locomotion (distance in $\mathrm{cm}$ ), counts of vertical movements, and stereotypies (repetitive beam breaks $<1 \mathrm{~s}$ apart). Location of mice was mapped and defined as being in either the center or perimeter zones (each $10.5 \mathrm{~cm}^{2}$ ). Data was recorded in 5 -min bins. Time-point values refer to total in each 5-min bin. OF data are reported during basal conditions (first $60 \mathrm{~min}$ in chamber) and following an injection of cocaine (20 mg/kg i.p.; minutes $61-180$ in chamber). The locomotor response to cocaine was normalized to the mean distance traveled during the 20 min immediately preceding cocaine administration ("baseline locomotion"). The genotype of the experimental subject was not known to the experimenter. Behavioral assays for each genotype were repeated in two separate cohorts. Transgenepositive (+/-) and transgene-negative (-/-) mice for each genotype were compared using 2-way repeated measures ANOVA.

\section{TISSUE FIXATION, IMMUNOSTAINING, AND FLUORESCENCE IMAGING}

Three-week-old mice were anesthetized with tribromoethanol (250 mg/kg i.p.) before transcardial perfusion with $4 \%$ paraformaldehyde (PFA) in phosphate-buffered saline (PBS), pH 7.4. Brains were extracted and immersed in PFA solution for 24-72 h at $4^{\circ} \mathrm{C}$ prior to the sectioning of $50 \mu \mathrm{m}$ slices on a Leica VTS- 1000 vibratome (Leica Microsystems, Bannockburn, IL, USA). Slices were transferred to $1 \mathrm{X}$ PBS for immunofluorescence staining of free floating sections except for slices prepared from line 6 Drd1atdTomato $^{+-} /$ChAT-EGFP ${ }^{+-}$mice which were directly mounted with Fluoromount-G mounting medium (Southern Biotech, Birmingham, AL, USA) without additional processing.

Slices were washed in PBS and incubated for $1 \mathrm{~h}$ in blocking buffer containing $5 \%$ bovine serum albumin, $2 \%$ normal goat serum, $0.2 \%$ Triton X-100 in $1 \mathrm{X}$ PBS prior to immunostaining. Slices were incubated overnight at $4^{\circ} \mathrm{C}$ with the following primary antibodies and dilutions: parvalbumin (PV) mouse monoclonal (P3088 Sigma, St. Louis, MO, USA) 1:500, somatostatin (SS) rat monoclonal (MAB354 Millipore, Billerica, MA 01821, USA) 1:50-1:100, and DARPP-32 rabbit polyclonal (AB1656 Millipore) 1:500. Secondary antibodies (Alexa Fluor-405 conjugated goat anti-mouse, Alexa Fluor-405 conjugated goat anti-rabbit, and Alexa Fluor-488 conjugated goat anti-mouse; all from Invitrogen, Carlsbad, CA, USA) 
were incubated at 1:800 dilutions. Cy5 conjugated donkey anti-rat antibodies were used at 1:200 dilution (Jackson ImmunoResearch Laboratories, Inc., Westgrove, PA, USA). Slices were mounted with Fluoromount-G mounting medium.

Sagittal slices were imaged on a Zeiss Axio Observer Z1 motorized microscope equipped with a $10 \times / 0.30$ Plan-NeoFluar Ph1 objective, X-CITE 120XL metal halide fluorescence light source, and Coolsnap ES2 high resolution CCD camera. TdTomato fluorescence was imaged with a red HQ45 filter cube (BP 560/40, FT 585, BP 630/75). The low-magnification sagittal brain view (Figure $3 \mathrm{~A}$ ) is a composite image created by stitching together multiple $10 \times$ images using MetaMorph 7.6.5 software (Molecular Devices, Sunnyvale, CA, USA). Higher resolution images were acquired using a Zeiss LSM 510 inverted confocal microscope equipped with $405 \mathrm{~nm}$ Diode, Argon/2 (458, 477, 488, 514 nm), 561 nm Diode and HeNe $633 \mathrm{~nm}$ lasers. Images were acquired using either $10 \times / 0.30 \mathrm{NA}$ (Plan Neo-Fluar) or 20×/0.80 NA (Plan Apochromat) objectives and acquisition software from Zeiss Microimaging (Thornwood, NY, USA). Confocal images were analyzed using ImageJ software (http://rsbweb.nih.gov/ij/).

Imaging of $300 \mu \mathrm{m}$ thick acute coronal cortical-striatal slices was performed on an electrophysiological rig with continuous perfusion of standard artificial cerebrospinal fluid (ACSF). Slices were prepared using the procedure described for electrophysiology recordings. Imaging was performed using a $60 \times$ water-immersion objective (LUMPlanFL N, 60×/1.00 NA) under an Olympus BX51WI microscope equipped with phase contrast optics, epifluorescence, an Olympus OLY-150 camera/controller system (Olympus America, Center Valley, PA, USA) and an HR-120 series monochrome video monitor (DAGE-MTI, Michigan City, IN, USA). Cells within the dorsal striatum were first identified by phase-contrast illumination and then checked for fluorescence. Cells that appeared swollen or otherwise necrotic were not included in the analysis.

For both confocal and epifluorescent imaging data, the threshold for a positive signal was an intensity equal to or greater than that of the surrounding neuropil. Cells lacking transgene fluorescence were defined as those in which fluorescence was significantly lower than the surrounding neuropil (characteristic "black hole" appearance). Note: these criteria are different from those used in our previous study to characterize the Drd1a-tdTomato line 5 mice, which required fluorescence intensity to be greater than surrounding neuropil to be considered positive (Shuen et al., 2008). To minimize light scattering effects which degrade imaging sensitivity, only cells in the first $60 \mu \mathrm{m}$ of tissue depth were assessed.

\section{ACUTE SLICE PREPARATION AND ELECTROPHYSIOLOGY}

For electrophysiological recordings, acute coronal brain slices (300 $\mu \mathrm{m}$ thick) were prepared from line 6 Drd1a-tdTomato ${ }^{+-} / \mathrm{Drd2}$ $\mathrm{EGFP}^{+/-}$transgenic mice and their line 6 Drd1a-tdTomato ${ }^{-1-} / \mathrm{Drd} 2-$ $\mathrm{EGFP}^{+/-}$transgenic littermates (P21-27). Mice were anesthetized with tribromoethanol $(250 \mathrm{mg} / \mathrm{kg}$ i.p. $)$ and transcardially perfused with ice-cold sucrose ACSF oxygenated with $95 \% \mathrm{O}_{2}$ and $5 \% \mathrm{CO}_{2}$ prior to decapitation. Brains were rapidly removed and sectioned in oxygenated, ice-cold sucrose ACSF with a Vibratome 1500 (Vibratome, Bannockburn, IL, USA). Sucrose ACSF contained (in mM): 203 sucrose, $2.5 \mathrm{KCl}, 1.2 \mathrm{NaH}_{2} \mathrm{PO}_{4}, 0.5 \mathrm{CaCl}_{2}, 10 \mathrm{MgCl}_{2}, 23 \mathrm{NaHCO}_{3}$, and $10 \mathrm{D}-(+)$-glucose. Slices were maintained in standard ACSF saturated with $95 \% \mathrm{O}_{2}$ and $5 \% \mathrm{CO}_{2}$ at room temperature $\left(24-25^{\circ} \mathrm{C}\right)$ for at least $1 \mathrm{~h}$ prior to use. Standard ACSF contained (in mM): $124 \mathrm{NaCl}, 2.5 \mathrm{KCl}$, 1.2 $\mathrm{NaH}_{2} \mathrm{PO}_{4}, 2 \mathrm{CaCl}_{2}, 1 \mathrm{MgCl}_{2}, 26 \mathrm{NaHCO}_{3}$, and $10 \mathrm{D}$-(+)-glucose.

Recordings were obtained at room temperature $\left(24-25^{\circ} \mathrm{C}\right)$ with continuous perfusion of standard ACSF at a rate of $2-3 \mathrm{ml} / \mathrm{min}$. Whole-cell, patch-clamp recordings from MSNs in the dorsolateral striatum were performed. Cells were visualized with a $40 \times$ waterimmersion objective (LUMPlanFI, $40 \times / 0.80 \mathrm{w}$ ) under an Olympus BX51WI microscope equipped with infrared differential interference contrast optics, epifluorescence and an Olympus OLY-150 camera/controller system (Olympus). Recording pipettes had a resistance of 2-3 M $\Omega$ when filled with internal pipette solution containing (in $\mathrm{mM}$ ): 120 potassium gluconate, $20 \mathrm{KCl}, 4 \mathrm{NaCl}, 10$ HEPES, 0.2 EGTA, 4 MgATP, $0.3 \mathrm{Na}_{2}$ GTP and 10 sodium phosphocreatine, $\mathrm{pH} 7.2-7.3$ and $\sim 300 \mathrm{mOsm}$. No compensation was made for the liquid junction potential. Signals were amplified with an Axopatch 200B amplifier (Molecular Devices), digitally converted with a Digidata 1440A analog-to-digital converter (Molecular Devices), and stored on a computer for subsequent off-line analysis.

After establishing whole-cell configuration, cells were voltage clamped at $-70 \mathrm{mV}$ to check for series resistance (Rs). Cells with Rs $<20 \mathrm{M} \Omega$ were then held in $I=0$ configuration and were allowed to equilibrate until the resting membrane potential (RMP) stabilized (approximately 3-5 min). The RMP was recorded for $30 \mathrm{~s}$ and mean was reported. Current-voltage relationship experiments (to evaluate input resistance and action potential firing rate) were initiated with a membrane potential of $-80 \mathrm{mV}$ and consisted of a series of current injections (500 ms duration) between -200 and $+400 \mathrm{pA}$ in $25 \mathrm{pA}$ delivered in step-wise increments. All current clamp recordings were sampled at $10 \mathrm{kHz}$ and low-pass filtered at $2 \mathrm{kHz}$. Electrophysiological traces were analyzed offline using Clampfit 10.0 (Molecular Devices). Rheobase for each cell was defined by the minimal current that evoked action potentials. Graphic presentation and statistical comparison of data were performed using GraphPad Prism software (La Jolla, CA, USA). The mean RMP and the rheobase for each cell were compared statistically across cell types using unpaired Student's $t$-tests. Two-way ANOVA was used to determine group effects of current injection and cell type on number of spikes and on membrane potential. Values are represented as mean \pm SEM.

\section{RESULTS}

\section{BEHAVIORAL TESTING}

We first investigated the integrity of behaviors that are sensitive to dopamine signaling including general locomotor activity and the acute response to cocaine (Chandler et al., 1990; Karasinska et al., 2005; Bateup et al., 2010). For these experiments, Drd1a-tdTomato line 6 mice were backcrossed for six generations to $\mathrm{C} 57 \mathrm{Bl} / 6$ mice to minimize behavioral variability due to hybrid strain background. When we compared hemizygous (+/-) mice to transgene-negative littermates $(-/-)$, we found that locomotor activity level was undisturbed in line 6 Drdla-tdTomato ${ }^{+-}$mice during OF testing (Figure 1A). In response to acute cocaine injection $(20 \mathrm{mg} / \mathrm{kg}$ i.p.), we observed a greater than three-fold increase in locomotor activity relative to the pre-injection baseline period in mice of both genotypes (Figure 1B). No differences in the acute locomotor response to cocaine were observed between line 6 Drd1a-tdTomato ${ }^{+-}$and their transgene-negative littermate controls (Figure 1B). 

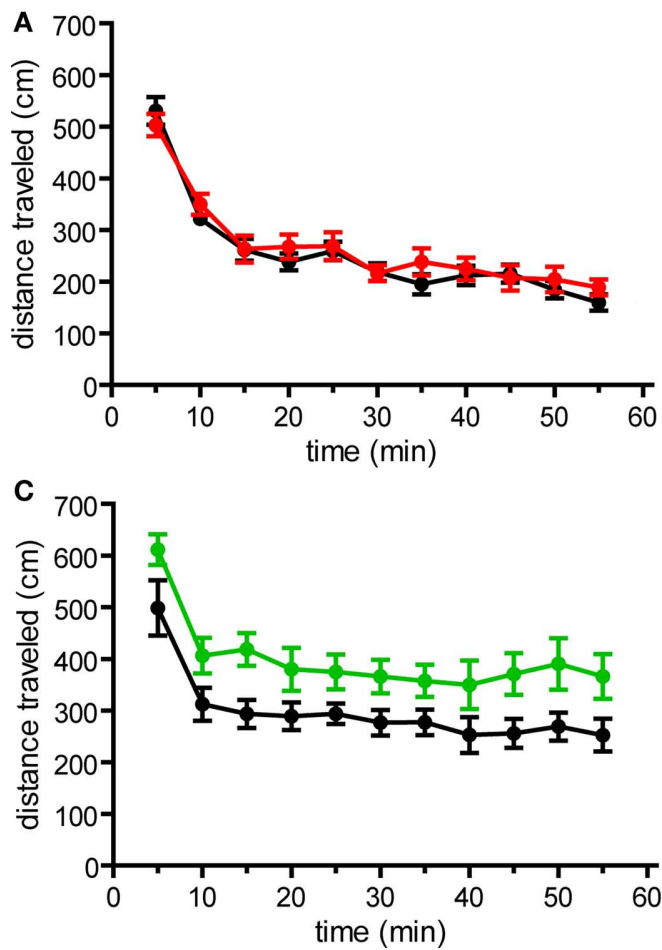

FIGURE 1 | Basal locomotor activity and acute locomotor response to cocaine in Drd1a-tdTomato line 6 and Drd2-EGFP BAC transgenic mice on C57BI/6 background. (A) Basal horizontal locomotion of Drd1atdTomato $^{+/-}$line 6 mice (red, $n=14$ ) is similar to transgene-negative (Drd1a-tdTomato-l-) littermates (black, $n=15 ; p=0.515$ ). (B) Acute cocaine administration (20 mg/kg i.p.) similarly increases horizontal locomotion in Drd1a-tdTomato ${ }^{+-}$mice (red, $n=14$ ) and Drd1a-tdTomato ${ }^{-/-}$littermates (black, $n=15 ; p=0.945)$. Blue trace indicates response to vehicle control injection
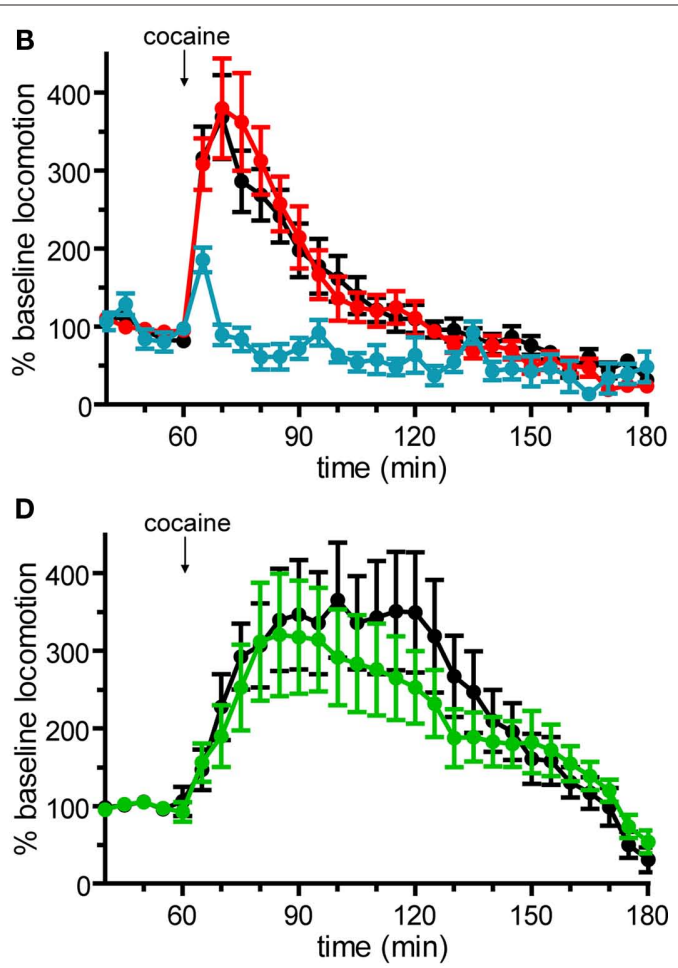

( $n=8$ mice, 4 each of Drd1a-tdTomato ${ }^{-/-}$and Drd1a-tdTomato ${ }^{+-}$mice. Responses were indistinguishable, so data were aggregated.) (C) Time course plots of basal horizontal locomotion demonstrate that Drd2-EGFP+/mice (green, $n=17$ ) are hyperactive compared to transgene-negative (Drd2-EGFP ${ }^{-1-}$ ) littermates (black, $n=15 ; p=0.027$ ). (D) Acute cocaine administration (20 mg/kg i.p.) induces a similar percent increase in locomotion in Drd2-EGFP ${ }^{+/}$mice (green, $n=17$ ) and Drd2-EGFP-/- littermates (black, $n=15 ; p=0.616)$
Basal locomotor behavior and acute locomotor response to cocaine were similarly tested in Drd2-EGFP transgenic mice. Drd2EGFP mice were first backcrossed to $\mathrm{C} 57 \mathrm{Bl} / 6$ mice for six generations. Behavioral testing of Drd2-EGFP/ C57BL/6 served three purposes in these experiments. First, if abnormal, these results would serve as a positive control for our ability to detect abnormalities in these behavioral domains. Second, to our knowledge, these experiments report the locomotor response to acute cocaine in hemizygous Drd2-EGFP transgenic mice for the first time. Third, by comparing $\operatorname{Drd} 2-\mathrm{EGFP}^{+/}$mice in a pure $\mathrm{C} 57 \mathrm{Bl} / 6$ background with their transgene-negative littermates, these experiments test for the first time the effects of the transgene on behavior in the absence of confounding variables which arise when using crossstrain behavioral controls. In contrast to line 6 mice, we found that basal locomotor activity during OF testing was significantly higher in hemizygous Drd2-EGFP mice as compared to their Drd2$\mathrm{EGFP}^{-/-}$littermates ( $p=0.027$, Figure 1C). Although hyperactive, no significant difference in the acute locomotor response to cocaine was observed (Figure 1D).

In addition to locomotor activity, other dopamine-dependent behavioral domains including vertical rearing, stereotypies and time spent in the center region of the OF chamber were assessed (Chandler et al., 1990; Tirelli and Witkin, 1994; Pogorelov et al., 2005). Neither line 6 nor Drd2-EGFP mice exhibited significant differences in vertical rearing or time spent performing stereotypic movements, either basally or in response to acute cocaine (Figures 2A-D). While line 6 mice showed no differences in the amount of time spent in the center region of the OF chamber (Figure 2E), Drd2-EGFP mice exhibited a statistically significant decrease in center time during basal activity $(p=0.013$, Figure $2 F)$. Neither line exhibited significant genotypic differences in center time during the response to acute cocaine injection ( $p=0.187$ for line 6 Drd1a-tdTomato and $p=0.535$ for Drd2-EGFP, data not shown). Finally, transgene-positive mice were indistinguishable from their transgene-negative littermate controls based on gross morphological features and body weight (Drd1atdTomato $^{+-} 22.9 \pm 1.1 \mathrm{~g}, n=15 ;$ Drd1a-tdTomato ${ }^{-1-} 22.0 \pm 1.0 \mathrm{~g}$, $n=14, p=0.601 ;$ Drd2-EGFP ${ }^{+/-} 19.8 \pm 0.4 \mathrm{~g}, n=17 ; \operatorname{Drd}_{2}-\mathrm{EGFP}^{-/}$ $20.3 \pm 0.7 \mathrm{~g}, n=15, p=0.524)$. In summary, these behavioral data demonstrate normal behavior in line 6 Drd1a-tdTomato ${ }^{+/-}$mice. These data also support the recently reported claim that the Drd2EGFP transgene may induce phenotypic alterations.

\section{SPECIFICITY OF tdTOMATO FLUORESCENCE}

Within the dorsal striatum of Drd1a-tdTomato line 6 mice, we next investigated the specificity of tdTomato fluorescence for correctly assigning a fluorescent MSN to the striatonigral pathway. 

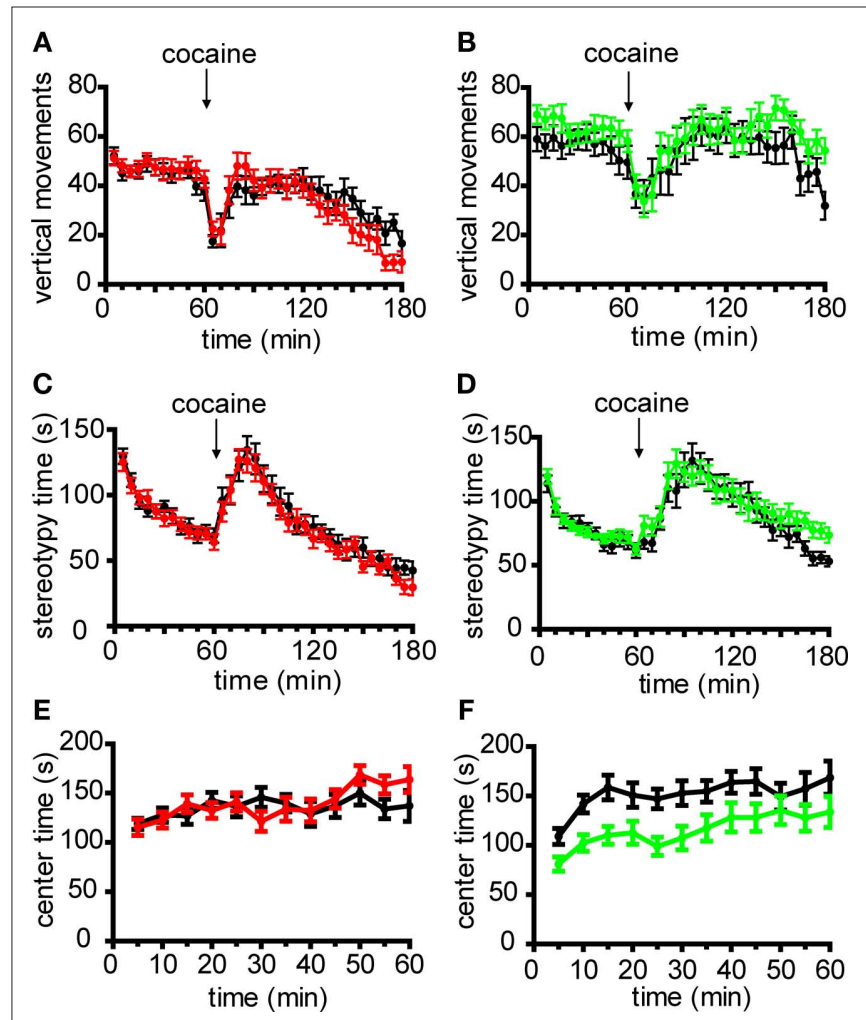

FIGURE 2 | Non-locomotor behaviors in line 6 Drd1a-tdTomato/C57BI/6 and Drd2-EGFP/C57BI/6 transgenic mice. Time course plots of the number of vertical movements basally and following acute cocaine injection $(20 \mathrm{mg} / \mathrm{kg}$ i.p.) demonstrate that transgene-positive mice are similar to transgenenegative littermate control mice in (A) Drd1a-tdTomato line 6 mice (Drd1atdTomato $^{+-}$, red, $n=14$; Drd1a-tdTomato ${ }^{-1}$, black, $n=15 ; p=0.778$ ) and (B) Drd2-EGFP mice (Drd2-EGFP+/, green, $n=17$; Drd2-EGFP ${ }^{-1-}$, black, $n=15$; $p=0.256)$. No differences in stereotypic movements basally and following acute cocaine injection were observed between transgene-positive and transgene-negative littermate control mice in (C) Drd1a-tdTomato line 6 mice (Drd1a-tdTomato ${ }^{+-}$, red, $n=14$; Drd1a-tdTomato ${ }^{-/}$, black, $n=15 ; p=0.615$ ) and (D) Drd2-EGFP mice (Drd2-EGFP+-, green, $n=17$; Drd2-EGFP--, black, $n=15 ; p=0.411$ ). (E) Drd1a-tdTomato line 6 mice and transgene-negative littermate control mice spend a similar amount of time in center region of open field test chamber (Drd1a-tdTomato ${ }^{+/}$, red, $n=14$; Drd1a-tdTomato ${ }^{-1}$, black, $n=15 ; p=0.647$ ). (F) Drd2-EGFP+/- (green, $n=17$ ) spend significantly less time in the center region of the open field chamber compared to Drd2-EGFP ${ }^{--}$littermates (black, $n=15 ; p=0.013$ ).

As expected for striatonigral MSNs, a low-magnification view of the brain from a Drd1a-tdTomato line 6 mouse demonstrates that fluorescent axonal projections from the striatum bypass the globus pallidus external (GPe) and terminate in the substantia nigra pars reticulata (SNr; Figure 3A). At the cellular level, we investigated the extent to which tdTomato expression could be segregated from EGFP expression in Drd1a-tdTomato ${ }^{+/} /$Drd2-EGFP ${ }^{+/-}$mice. In mice, the vast majority of striatal MSNs send axons through either the striatonigral or striatopallidal pathway, and these two populations of MSN projection neurons can be discriminated by expression of the type 1 and type 2 dopamine receptors, respectively (Gerfen et al., 1990). Thus, if the transgenes reliably identify these two populations, it is predicted that there should not be significant co-expression of the fluorescent reporters among striatal projection neurons. As predicted, the striatal MSNs identified by DARPP-32 immunolabeling (Anderson and Reiner, 1991; Ouimet et al., 1998) with detectable levels of both EGFP and tdTomato fluorescence were very rare (2.3\% of 1095 DARPP-32 positive cells; Figure 3B).

Besides MSNs, interneurons of various types collectively represent approximately $5 \%$ of striatal neurons (see review by Tepper et al., 2010). Expression of tdTomato was investigated in the three most abundant interneuronal types - fast-spiking interneurons (FSIs), low-threshold spiking interneurons (LTS interneurons), and large tonically active aspiny cholinergic interneurons (TANs). PV immunostaining, to identify FSIs, failed to identify any FSIs with fluorescence from either the Drd1a-tdTomato (Figure 3C) or Drd2-EGFP transgenes (0/52 PV-positive cells). Likewise, somatostatin immunostaining, to identify LTS interneurons, demonstrated that these cells do not contain detectable levels of fluorescence due to either the Drd1a-tdTomato (Figure 3D) or Drd2-EGFP transgenes (0/45 somatostatin-positive cells). TANs were visualized by EGFP fluorescence in ChAT-EGFP transgenic mice. No overlap was observed between tdTomato and EGFP fluorescence in slices prepared from Drdla-tdTomato ${ }^{+/} /$ChAT$\mathrm{EGFP}^{+/-}$mice (0/56 EGFP-fluorescent cells, Figure 3E). Lastly, astrocytes, as indicated by immunostaining with glial fibrillary acidic protein (GFAP), did not appreciably express tdTomato $(0 / 37$ GFAP-positive cells, data not shown). In summary, these studies indicate that tdTomato fluorescence in dorsal striatal cells is highly specific to striatonigral MSNs.

\section{SENSITIVITY OF tdTOMATO FLUORESCENCE}

We next investigated whether tdTomato fluorescence also provided adequate sensitivity for detecting the majority of striatonigral MSNs. We addressed this question using two imaging approaches, confocal microscopy of thin fixed brain sections and epifluorescent microscopy of thicker acute brain slices (as commonly prepared for electrophysiological experiments), to define the upper and lower estimates of fluorescence detection sensitivity. In confocal imaging experiments, MSNs were defined by DARPP-32 immunostaining. In mice that were hemizygous for both Drd1a-tdTomato and Drd2EGFP transgenes, 8 of 1095 DARPP-32-positive cells $(0.7 \%)$ did not have detectable fluorescence from either the tdTomato or EGFP reporter proteins (Figure 3B). This finding demonstrates that both transgenic reporters have negligible rates of false negativity for identifying MSNs under these imaging conditions (for details see Materials and Methods). We next examined the sensitivity of fluorescence detection in MSNs under imaging conditions commonly used for identification of cells in live tissue during electrophysiological experiments. In acute brain slices ( $300 \mu \mathrm{m}$ thick), mediumsized cells were first identified using phase-contrast illumination and then evaluated for fluorescence. Again, the vast majority of cells were accounted for by detectable fluorescence from the transgenic reporter proteins ( $518 / 544$ cells; $>95 \%$, Figure $3 B$ ). These findings indicate that tdTomato fluorescence in line 6 mice can be highly sensitive and suitable for identifying all striatonigral MSNs.

\section{INTEGRITY OF tdTOMATO-EXPRESSING MSNs}

We considered whether the ectopic expression of tdTomato proteins in MSNs might alter cellular health (Vintersten et al., 2004; Long et al., 2005). We did not find evidence to support a toxic 
A

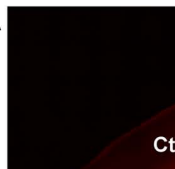

Ctx

DS
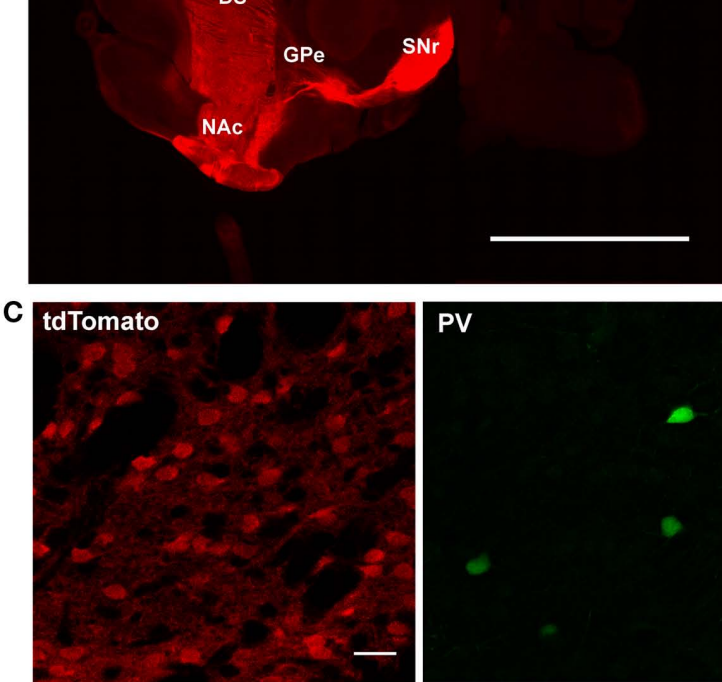

D tdTomato

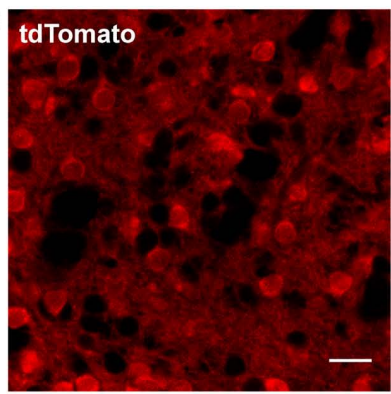

E

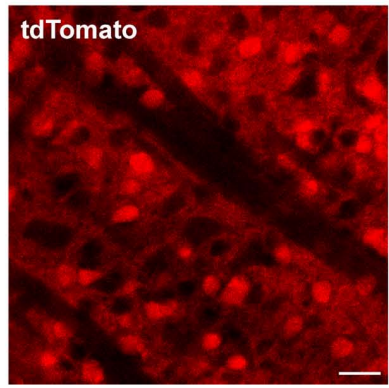

PV

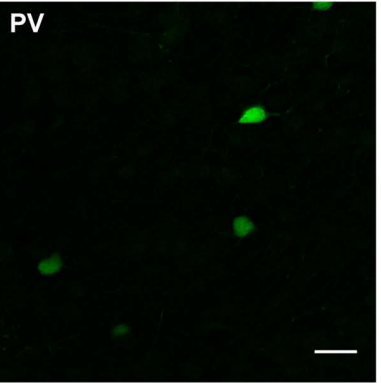

SS

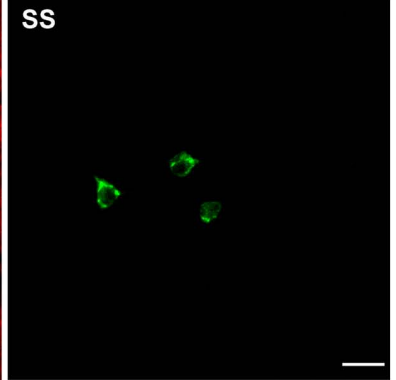

ChAT-EGFP

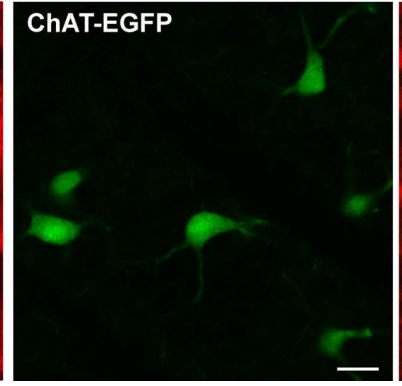

B

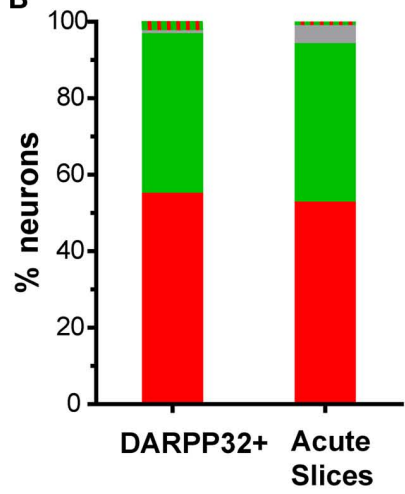

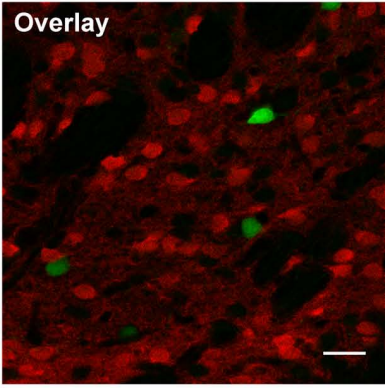
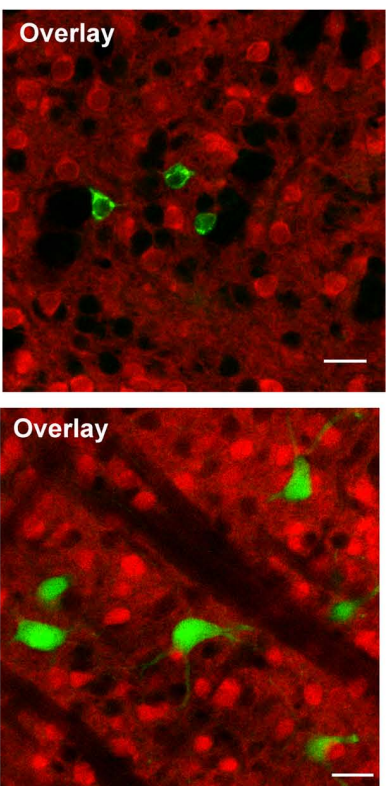

FIGURE 3 |TdTomato fluorescence is sensitive and specific for striatonigral MSNs in the dorsal striatum. (A) A representative low-magnification sagittal view of tdTomato fluorescence of a brain slice from a hemizygous line 6 Drd1a-tdTomato mouse demonstrating strong signal within striatal efferent axons terminating in substantia nigra pars reticulata ( $\mathrm{SNr}$ ) and negligible signal at the globus pallidus external (GPe). Abbreviations: cortex (Ctx), cerebellum (CB), dorsal striatum (DS) and nucleus accumbens (NAc). Scale bar represents $500 \mu \mathrm{m}$. (B) Quantification of fluorescent signal detected in dorsal striatal cells from line 6 Drd1a-tdTomato+//Drd2-EGFP+/- mice. DARPP32+ cells were identified in immunohistochemically stained $50 \mu \mathrm{m}$ thick brain sections $(n=605$ tdTomato only (red), 457 EGFP only (green), 25 dually labeled (red and green striped), and 8 non-fluorescent (gray) of 1095 total DARPP-32 immunopositive cells). Cells in acute $300 \mu \mathrm{m}$ thick slices were identified by phase-contrast illumination. Fluorescent signals were detected using epifluorescence illumination ( $n=293$ tdTomato only (red), 230 EGFP only (green), 5 dually labeled (red and green striped), and 26 non-fluorescent (gray) of 549 total medium size cells identified by phase contrast illumination). (C) Representative confocal microscopy images demonstrating that cells immunopositive for the fast-spiking GABAergic interneuron marker, parvalbumin (PV), do not express tdTomato $(0 / 52$ PV-immunopositive cells). (D) Representative confocal microscopy images demonstrating cells immunopositive for the low-threshold spiking GABAergic interneuron marker, somatostatin (SS), do not express tdTomato (0/45

SS-immunopositive cells). (E) Representative confocal microscopy images demonstrating no overlap between tdTomato and EGFP fluorescence in line 6 Drd1a-tdTomato+//ChAT-EGFP+- mice (0/56 EGFP-positive cells). For panels (C-E), tdTomato fluorescence (left), interneuron antigen or transgenic reporter fluorescence (middle) and overlay of both signals (right). Scale bars represent $25 \mu \mathrm{m}$. 
effect resulting in the loss of striatonigral MSNs because the relative fraction of the total MSNs accounted for by tdTomato-positive neurons (57.9\%, Figure 3B) was similar to what has been previously reported for striatonigral neurons (Bertran-Gonzalez et al., 2008). To further test cellular integrity, we compared the physiological properties of tdTomato-fluorescent neurons from Drd1atdTomato $^{+/-} /$Drd2-EGFP ${ }^{+/-}$mice to non-fluorescent neurons from Drd1a-tdTomato ${ }^{-/-} /$Drd2-EGFP ${ }^{+/-}$littermate control mice. We found no significant differences in RMP, membrane input resistance, rheobase or action potential firing rates (Figure 4).

\section{DISCUSSION}

In this study, we characterize a new Drd1a-tdTomato BAC transgenic mouse line, line 6 . Our results show that line 6 mice provide both high specificity and sensitivity for the identification of striatonigral (direct) pathway MSNs and offer several advantages over existing transgenic mouse lines that label striatal projection pathways. Foremost, recent concerns about phenotypic alteration of mice in a commonly used Drd2-EGFP transgenic line have raised a need to find alternative means for identifying direct and indirect pathway MSNs. Our results indicate that line 6 mice do not have phenotypic alterations and, if used under experimental conditions similar to those we report here, can be used as a single transgenic line to accurately assign MSNs to both pathways based on the presence or absence of fluorescence. Drd1a-tdTomato line 6 mice also offer the advantages that are afforded by (1) the brighter fluorescence of tdTomato in comparison to EGFP (Deliolanis et al., 2008; Drobizhev et al., 2009) and to line 5 Drdla-tdTomato mice (data not shown), (2) an excitation wavelength which provides deeper tissue penetration (reduced scatter) than the excitation wavelength used for EGFP, (3) autosomal inheritance of the transgene, and (4) the ability to easily maintain on a $\mathrm{C} 57 \mathrm{Bl} / 6$ background. Although the present study focuses on tdTomato expression in the dorsolateral striatum, representative images of other brain regions that may be of interest are provided (Figure 5).

In this study, we first investigated the behavioral integrity of line 6 mice and found no evidence for behavioral abnormalities in activity basally or in response to cocaine during OF testing. These results are similar to those reported for another line of transgenic mice that also contain the upstream regulatory elements for Drd1a, GENSAT Drd1a-EGFP mice (Kramer et al., 2011). Although the focus of our study was to characterize the new transgenic line, line 6 Drd1a-tdTomato, we also provide new evidence that supports the findings by Alvarez and colleagues that Drd2-EGFP transgenic mice have altered behavioral phenotypes (Kramer et al., 2011). Both studies demonstrate increased basal locomotion in Drd2-EGFP mice, although to differing degrees. In this study, we found that hemizygous mice on a C57Bl/6 background exhibit approximately $130 \%$ of littermate control activity levels. In the recent report by Alvarez and colleagues, homozygous mice on a hybrid background exhibited approximately 300\% of Swiss Webster control activity levels (Kramer et al., 2011). Following acute cocaine challenge (20 mg/kg i.p.), we observed a robust increase in locomotor activity by hemizygous $\mathrm{Drd2}$-EGFP/C57Bl/6 mice that was similar to littermate controls. The degree of acute cocaine-induced locomotor response we observe is consistent with prior observations in C57Bl/6 mice (Schlussman et al., 2003). Testing homozygous

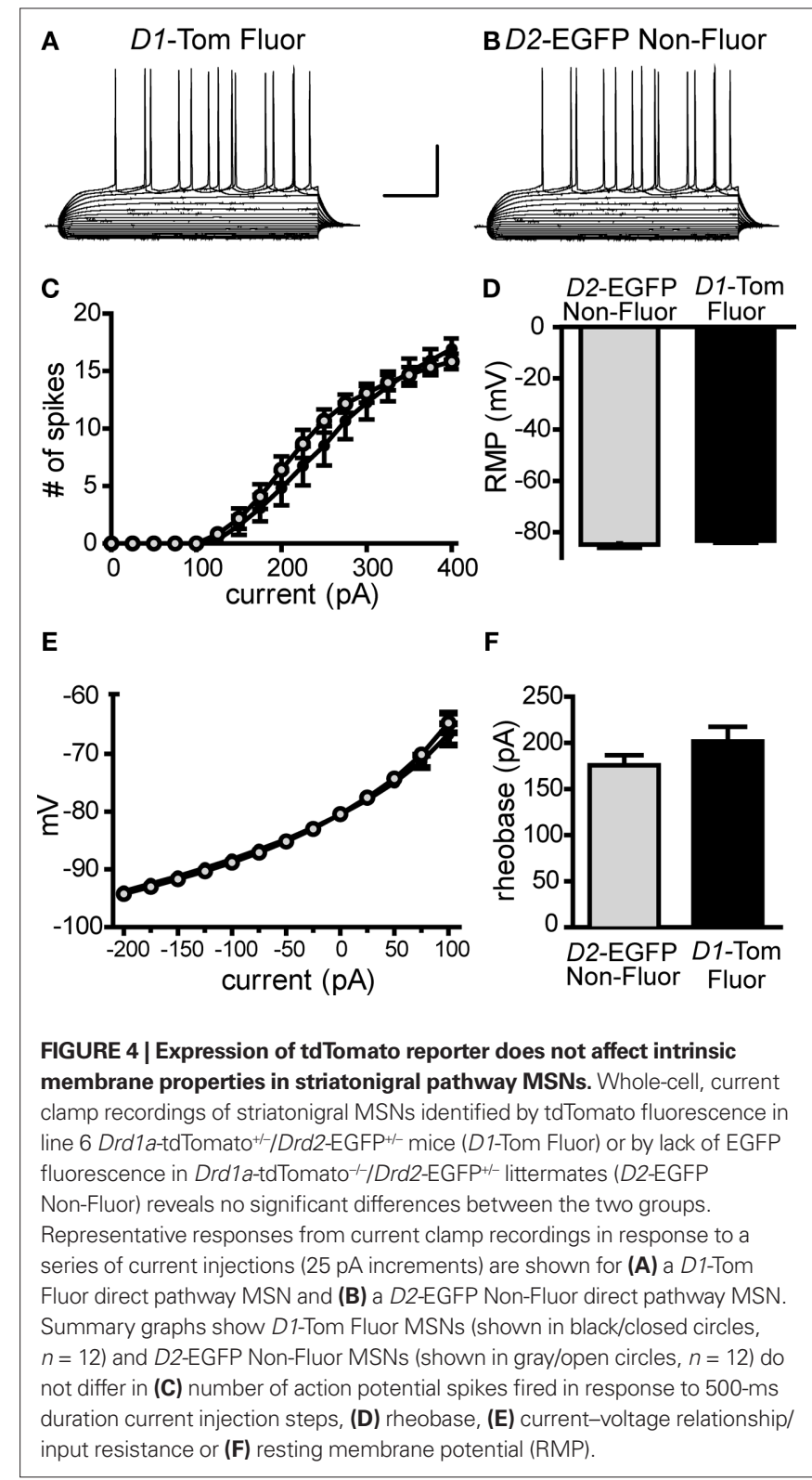

Drd2-EGFP mice, Kramer et al. found that there was no significant locomotor response to acute cocaine when compared to injection with saline. It is presently unclear if the genotype (hemi v. homo), strain background (C57Bl/6 v. hybrid) or other experimental design features are responsible for this difference. However, it is notable that, in the present study, we control for strain background, test only hemizygous mice, and use transgene-negative littermate controls and still find that in two assays (basal levels of locomotion and time spent in center of test chamber) Drd2-EGFP transgenic animals have altered behavior. Thus, we provide further evidence that the Drd2-EGFP transgenic mouse line has unintended phenotypic disturbances.

Due to concerns about phenotypic abnormalities in the Drd2EGFP line, alternative methods to identify striatopallidal pathway neurons are desirable. The GENSAT A2A-EGFP mice (Gong et al., 2003) provide one such alternative (Warre et al., 2011), although, 


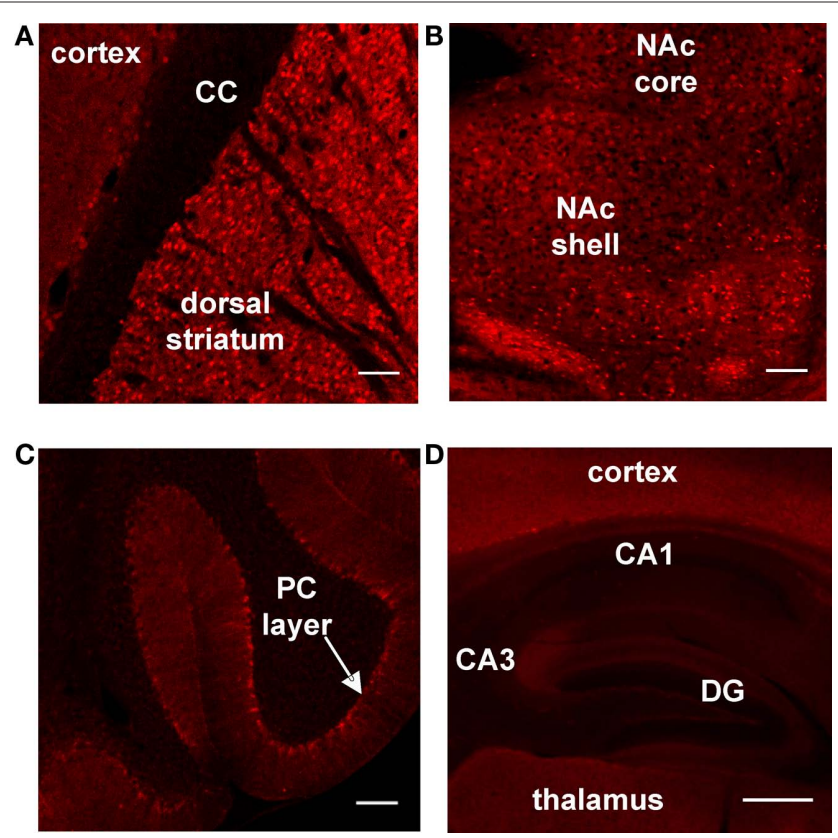

FIGURE 5 | Survey of tdTomato expression in sagittal brain slices prepared from line 6 Drd1a-tdTomato ${ }^{+/}$mice. Representative confocal images showing tdTomato expression at (A) the border between cortex and striatum (corpus callosum = CC), (B) the nucleus accumbens (NAc) and (C) the cerebellum (Purkinje cell = PC). (D) Representative epifluorescent image showing tdTomato expression in the hippocampus (dentate gyrus = DG). The contrast has been enhanced for each image to highlight tdTomato expression patterns within the subregion. Scale bars for all panels represent $100 \mu \mathrm{m}$.

behavioral characterization of these mice has not yet been reported. Another alternative is to use non-fluorescent cells in a line that reports direct pathway MSNs with high specificity and sensitivity. The results of the present study indicate that assignment of nonfluorescent cells to the indirect pathway can be highly accurate in line 6 mice (Figure 3B). In mice expressing both the Drd2-EGFP and Drd1a-tdTomato transgenes, greater than $98 \%$ of MSNs lacking tdTomato fluorescence had detectable EGFP fluorescence under sensitive confocal imaging conditions. Under less sensitive conditions (epifluorescent imaging and thicker acute brain slices), we still found that, among cells identified by phase contrast illumination, $>90 \%$ of the cells lacking tdTomato fluorescence had EGFP fluorescence. Therefore, classifying indirect pathway MSNs solely by the absence of tdTomato fluorescence (without taking advantage of additional means to define a cell as a MSN), one can still

\section{REFERENCES}

Ade, K. K., Janssen, M. J., Ortinski, P. I., and Vicini, S. (2008). Differential tonic GABA conductances in striatal medium spiny neurons. J. Neurosci. 28 , 1185-1197.

Anderson, K. D., and Reiner, A. (1991). Immunohistochemical localization of DARPP-32 in striatal projection neurons and striatal interneurons: implications for the localization of
D1-like dopamine receptors on different types of striatal neurons. Brain Res. 568, 235-243.

André,V.M.,Cepeda,C., Fisher,Y.E.,Huynh, M., Bardakjian, N., Singh, S., Yang,X.W., and Levine, M. S. (2011). Differential electrophysiological changes in striatal output neurons in Huntington's disease. J. Neurosci. 31, 1170-1182.

Bateup, H. S., Santini, E., Shen, W., Birnbaum, S., Valjent, E., Surmeier,

expect less than $10 \%$ contamination of indirect pathway data sets by type I errors. In reality, soma appearance and electrophysiological characteristics are readily available to the electrophysiologist allowing them to restrict analyses to the subset of cells that are MSNs. We also characterized tdTomato fluorescence in striatal interneurons. Collectively, interneurons comprise approximately $5 \%$ of total striatal neurons (Tepper et al., 2010). No tdTomato fluorescence was detected in the three most abundant striatal interneuron subtypes - tonically active cholinergic interneurons, fast-spiking GABAergic interneurons and low-threshold spiking interneurons. Other sparser interneuronal populations, such as calretinin positive GABArgic inteneurons and tyrosine hydroxylase positive interneurons were not evaluated, and therefore, may express tdTomato. Finally, among a total of 2373 fluorescent cells from Drd1a-tdTomato ${ }^{+/} /$Drd2-EGFP ${ }^{+/-}$mice, only $1.6 \%$ of cells expressed detectable levels of both fluorophores.

\section{CONCLUSION}

In summary, this study characterizes a new transgenic line that expresses tdTomato under the regulatory elements of the Drdla gene. In the experiments reported herein, we found no evidence for changes in behavior or basic cellular properties due to transgene expression in Drd1a-tdTomato line 6 mice. Furthermore, the high selectivity and sensitivity of tdTomato fluorescence for reporting striatonigral MSNs in line 6 mice provides a reliable method for classifying both direct and indirect pathway neurons using a single transgenic mouse line.

\section{ACKNOWLEDGMENTS}

We would like to acknowledge the following sources for financial support: National Institute of Neurological Disorders and Stroke [T32NS051156 (Kristen Ade), NS064577 (Nicole Calakos), NS064577-S1 (Nicole Calakos), NS054840 (Nicole Calakos)], Klingenstein Foundation (Nicole Calakos), NARSAD (Nicole Calakos) and Duke Institute for Brain Sciences (Nicole Calakos). We would like to thank Samantha Tracy for excellent care and management of mouse colonies; Drs. Jei Deng and Anne West for consultation and reagents used in immunostaining experiments; Dr. Gregg Stanwood for noting the X-linked inheritance pattern of line 5 mice; Drs. Sam Johnson and Yasheng Gao of the Duke University Light Microscopy Core Facility for imaging equipment and consultation; and Drs. William Wetsel and Ramona Rodriguiz and the Neurobehavioral Core Facility for consultation and assistance with behavioral experiments. We thank members of our laboratory and Dr. Jonathan Ting for critical discussion and manuscript comments.

D. J., Fisone, G., Nestler, E. J., and Greengard, P. (2010). Distinct subclasses of medium spiny neurons differentially regulate striatal motor behaviors. Proc. Natl. Acad. Sci. U.S.A. 107, 14845-14850.

Bertran-Gonzalez, J., Bosch, C., Maroteaux, M., Matamales, M., Herve, D., Valjent, E., and Girault, J.A. (2008). Opposing patterns of signaling activation in dopamine D1 and D2 receptor- expressing striatal neurons in response to cocaine and haloperidol. J. Neurosci. 28, 5671-5685.

Cepeda, C., Andre, V. M., Yamazaki, I. Wu, N., Kleiman-Weiner, M., and Levine, M. S. (2008). Differential electrophysiological properties of dopamine D1 and D2 receptorcontaining striatal medium-sized spiny neurons. Eur. J. Neurosci. 27, 671-682. 
Chandler, C. J., Starr, B. S., and Starr, M. S. (1990). Differential behavioural interactions between the dopamine D-1 antagonist SCH 23390 and the dopamine D-2 antagonists metoclopramide and sulpiride in nonhabituated mice. Pharmacol. Biochem. Behav. 35, 285-289.

Day, M., Wang, Z., Ding, J., An, X., Ingham, C.A., Shering, A. F., Wokosin, D., Ilijic, E., Sun, Z., Sampson, A. R., Mugnaini, E., Deutch, A. Y., Sesack, S. R., Arbuthnott, G. W., and Surmeier, D. J. (2006). Selective elimination of glutamatergic synapses on striatopallidal neurons in Parkinson disease models. Nat. Neurosci. 9, 251-259.

Day, M., Wokosin, D., Plotkin, J. L., Tian, X., and Surmeier, D. J. (2008). Differential excitability and modulation of striatal medium spiny neuron dendrites. J. Neurosci. 28, 11603-11614.

Deliolanis, N. C., Kasmieh, R., Wurdinger, T., Tannous, B. A., Shah, K., and Ntziachristos, V. (2008). Performance of the red-shifted fluorescent proteins in deep-tissue molecular imaging applications. J. Biomed. Opt. 13, 044008.

Doig, N. M., Moss, J., and Bolam, J. P. (2010). Cortical and thalamic innervation of direct and indirect pathway medium-sized spiny neurons in mouse striatum. J. Neurosci. 30 14610-14618.

Drobizhev, M., Tillo, S., Makarov, N. S., Hughes, T. E., and Rebane, A. (2009). Absolute two-photon absorption spectra and two-photon brightness of orange and red fluorescent proteins. $J$. Phys. Chem. B 113, 855-859.

Flores-Barrera, E., Vizcarra-Chacon, B. J., Tapia, D., Bargas, J., and Galarraga, E. (2010). Different corticostriatal integration in spiny projection neurons from direct and indirect pathways. Front. Syst. Neurosci. 4:15. doi: 10.3389/fnsys.2010.00015

Gerfen, C. R., Engber, T. M., Mahan, L. C., Susel, Z., Chase, T. N., Monsma, F. J., Jr., and Sibley, D. R. (1990). D1 and D2 dopamine receptor-regulated gene expression of striatonigral and striatopallidal neurons. Science 250, 1429-1432.

Gertler, T. S., Chan, C. S., and Surmeier D. J. (2008). Dichotomous anatomical properties of adult striatal medium spiny neurons. J. Neurosci. 28, 10814-10824.

Gittis, A. H., Nelson, A. B., Thwin, M. T., Palop, J. J., and Kreitzer, A. C. (2010). Distinct roles of GABAergic interneurons in the regulation of striatal output pathways. J. Neurosci. 30, 2223-2234.

Gong, S., Zheng, C., Doughty, M. L., Losos, K., Didkovsky, N., Schambra, U. B., Nowak, N. J., Joyner, A., Leblanc, G., Hatten, M. E., and Heintz, N. (2003). A gene expression atlas of the central nervous system based on bacterial artificial chromosomes. Nature 425, 917-925.

Janssen, M. J., Ade, K. K., Fu, Z., and Vicini, S. (2009). Dopamine modulation of GABA tonic conductance in striatal output neurons. J. Neurosci. 29, 5116-5126.

Karasinska, J. M., George, S. R., Cheng, R., and O'Dowd, B. F. (2005). Deletion of dopamine $\mathrm{D} 1$ and $\mathrm{D} 3$ receptors differentially affects spontaneous behaviour and cocaine-induced locomotor activity, reward and CREB phosphorylation. Eur. J. Neurosci. 22, 1741-1750.

Kramer, P. F., Christensen, C. H., Hazelwood, L. A., Dobi, A., Bock, R., Sibley, D. R., Mateo, Y., and Alvarez, V. A. (2011). Dopamine D2 receptor overexpression alters behavior and physiology in Drd2-EGFP mice. $J$. Neurosci. 31, 126-132.

Kreitzer,A.C., and Malenka, R. C. (2007) Endocannabinoid-mediated rescue of striatal LTD and motor deficits in Parkinson's disease models. Nature 445, 643-647.

Long, J. Z., Lackan, C. S., and Hadjantonakis, A. K. (2005). Genetic and spectrally distinct in vivo imaging: embryonic stem cells and mice with widespread expression of a monomeric red fluorescent protein. BMC Biotechnol. 5, 20. doi: 10.1186/1472-6750-5-20
Ouimet, C. C., Langley-Gullion, K. C. and Greengard, P. (1998). Quantitative immunocytochemistry of DARPP-32expressing neurons in the rat caudatoputamen. Brain Res. 808, 8-12.

Planert, H., Szydlowski, S. N., Hjorth, J. J. Grillner, S., and Silberberg, G. (2010). Dynamics of synaptic transmission between fast-spiking interneurons and striatal projection neurons of the direct and indirect pathways. $J$. Neurosci. 30, 3499-3507.

Pogorelov, V. M., Rodriguiz, R. M., Insco, M. L., Caron, M. G., and Wetsel, W C. (2005). Novelty seeking and stereotypic activation of behavior in mice with disruption of the Dat 1 gene. Neuropsychopharmacology 30, 1818-1831.

Schlussman, S. D., Zhang, Y., Kane, S., Stewart, C. L., Ho, A., and Kreek, M. J. (2003).Locomotion, stereotypy, and dopamine D1 receptors after chronic "binge" cocaine in C57BL/6 J and 129/J mice. Pharmacol. Biochem. Behav. 75 123-131.

Shen, W., Flajolet, M., Greengard, P., and Surmeier, D. J. (2008). Dichotomous dopaminergic control of striatal synaptic plasticity. Science 321, 848-851.

Shen, W., Tian, X., Day, M., Ulrich, S. Tkatch, T., Nathanson, N. M., and Surmeier, D. J. (2007). Cholinergic modulation of Kir2 channels selectively elevates dendritic excitability in striatopallidal neurons. Nat. Neurosci. 10, 1458-1466.

Shuen, J. A., Chen, M., Gloss, B., and Calakos, N. (2008). Drdla-tdTomato BAC transgenic mice for simultaneous visualization of medium spiny neurons in the direct and indirect pathways of the basal ganglia. J. Neurosci. 28, 2681-2685.

Tepper, J. M., Tecuapetla, F., Koos, T. and Ibanez-Sandoval, O. (2010). Heterogeneity and diversity of striatal GABAergic interneurons. Front Neuroanat. 4:150. doi: 10.3389/ fnana.2010.00150

Tirelli, E., and Witkin, J. M. (1994). Verticalization of behavior elicited by dopaminergic mobilization is qualitatively different between C57BL/6J and DBA/2J mice. Psychopharmacology (Berl.) 116, 191-200.

Vintersten, K., Monetti, C., Gertsenstein, M., Zhang, P., Laszlo, L., Biechele, S. and Nagy, A. (2004). Mouse in red: red fluorescent protein expression in mouse ES cells, embryos, and adult animals. Genesis 40, 241-246.

Warre, R., Thiele, S., Talwar, S., Kamal, M., Johnston, T. H., Wang, S., Lam, D., Lo, C., Khademullah, C. S., Perera, G., Reyes, G., Sun, X. S., Brotchie, J.M., and Nash, J. E. (2011). Altered function of glutamatergic cortico-striatal synapses causes output pathway abnormalities in a chronic model of Parkinsonism. Neurobiol. Dis. 41, 591-604.

Yin, H. H., Mulcare, S. P., Hilario, M. R. Clouse, E., Holloway, T., Davis, M. I., Hansson, A. C., Lovinger, D. M., and Costa, R. M. (2009). Dynamic reorganization of striatal circuits during the acquisition and consolidation of a skill. Nat. Neurosci. 12,333-341.

Conflict of Interest Statement: The authors declare that the research was conducted in the absence of any commercial or financial relationships that could be construed as a potential conflict of interest.

Received: 30 March 2011; paper pending published: 13 April 2011; accepted:09 May 2011; published online: 08 June 2011.

Citation: Ade KK, Wan Y, Chen M, Gloss $B$ and Calakos N (2011) An improved $B A C$ transgenic fluorescent reporter line for sensitive and specific identification of striatonigral medium spiny neurons. Front. Syst. Neurosci. 5:32. doi: 10.3389/ fnsys.2011.00032

Copyright (C) 2011 Ade, Wan, Chen, Gloss and Calakos. This is an open-access article subject to a non-exclusive license between the authors and Frontiers Media $S A$, which permits use, distribution and reproduction in other forums, provided the original authors and source are credited and other Frontiers conditions are complied with. 